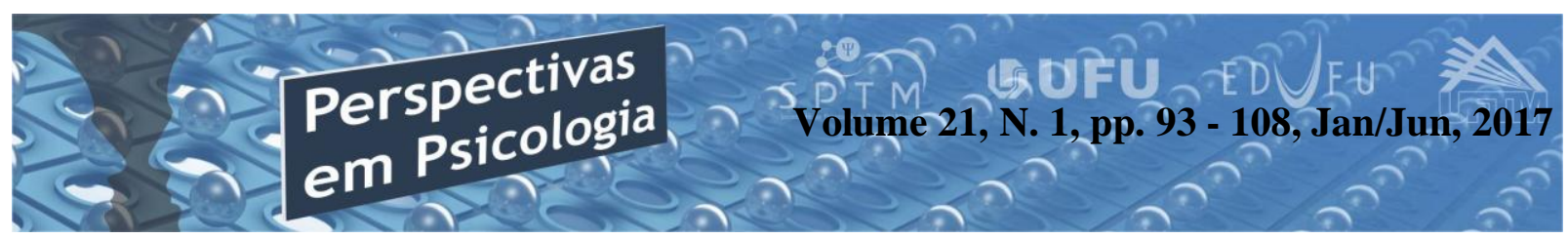

\title{
ÉTICA NA PESQUISA CIENTÍFICA: REVISÃO INTEGRATIVA
}

\author{
Fabiana Divina da Silva \\ Roberta Almeida Elias \\ Sulamita da Silva Lucas \\ Roselma Lucchese \\ Ivânia Vera \\ (Universidade Federal de Goiás, Regional Catalão - UFG/RC)
}

\begin{abstract}
Resumo
Este estudo objetivou sistematizar o conhecimento produzido acerca da integridade científica no cenário atual. Para tanto, realizou-se uma revisão integrativa, nas bases de dados eletrônicos: Literatura Latino-Americana e do Caribe em Ciências da Saúde (LILACS), Coordenação de Aperfeiçoamento de Pessoal de Nível Superior (CAPES), Scientific Electronic Library Online (Scielo) e National Library of Medicine - National Institutes of Health (NLMNIH). Utilizando-se dos descritores controlados plagiarism AND scientific misconduct and ethics research, nos idiomas português, inglês e espanhol, entre 2006 e 2016. Foram selecionados 9 artigos, todos com evidências de estudo não experimentais, qualitativas ou estudos de casos. Identifica-se nas publicações, uma preocupação com a má conduta científica, em destaque ao plágio e uma embrionária discussão sobre o tema prevenção e punição.
\end{abstract}

Palavras-chave: ética em pesquisa; plágio; má conduta científica.

\section{Abstract \\ Ethics in Scientific Research: Integration Review}

The study aims to systematize the knowledge produced about scientific integrity in the current scenario. An integrative review was held in electronic databases: Latin American literature and Caribbean Health Sciences (LILACS), Coordenação de Aperfeiçoamento de Pessoal de Nível Superior (CAPES), Scientific Electronic Library Online (Scielo) and National Library of Medicine-Health National Institutesof (NLMNIH). Using the controlled descriptors plagiarism AND scientific misconduct AND ethics research, in Portuguese, English and Spanish languages, between 2006 and 2016. 9 articles were selected, all with evidence of nonexperimental, qualitative study or case studies. Identifies the publications, a concern with scientific misconduct, plagiarism and featured an early discussion on the topic prevention and punishment.

Keywords: ethics research; plagiarism; scientific misconduct. 


\section{Introdução}

O cenário da produção de conhecimento científico passou por várias mudanças de paradigmas de publicações (Cury \& Rösing, 2013) e a preocupação com a integridade cientifica não é um problema enfrentado somente por líderes mundiais de publicações científicas, países emergentes em produção de ciência como o Brasil, juntam-se a esse problema de escala global (Alisson, 2014).

Entende-se que, a comunidade científica já tem esclarecido a síntese dos conceitos que devem compor a autoria do produto científico (Cury \& Rösing, 2013). No entanto para este estudo, o termo integridade científica se refere aos valores absolutos de honestidade, objetividade, veracidade, justiça, beneficência e responsabilidade do pesquisador (Pithan $\&$ Oliveira, 2013; FAPESP, 2014).

A integridade na pesquisa científica tem como objetivo garantir que as práticas éticas como a honestidade, prudência e reconhecimento de igualdade sejam adotadas pelos pesquisadores dentro das instituições, estando atrelados a qualquer pesquisa desenvolvida, seja uma produção científica ou intelectual. Os resultados destas pesquisas tornam-se o produto científico, que tem impulsionado a economia, e se tornam uma moeda mundial (Russo, 2014).

Assim sendo, um estudo sobre o tema integridade científica é essencial para que autores e editores adotem definiç̧ões de padrões éticos e práticas delimitadas, para assim garantir não só a quantidade, mas também a qualidade das publicações (Marques, 2014).

Mesmo que se tenha ciência, e a comunidade acadêmica conheça os conceitos de elementos necessários para produção científica, ainda se faz necessário elucidar e popularizar termos considerados clássicos nessa temática e que não possuem regras muito claras, como (auto) plágio, falsificação, fabricação e duplicação de publicação (Panter, 2015).

Ademais, há de se harmonizar as várias concepções existentes sobre má conduta científica, uma vez que se convive com enfoques individuais em cada país, com diversidade de aspecto das facetas do leque que se abre sobre a questão da integridade científica, que julgam ser mais importante ressaltar (Marques, 2014).

Diante do exposto, conhecer a linguagem utilizada no contexto mundial, acerca das impressões sobre a má conduta científica faz com quese contribua com a ampliação da qualidade da produção científica. Desse modo, esta pesquisa tem 
por objetivo sistematizar o conhecimento produzido acerca da integridade científica no cenário atual.

\section{Metodologia}

No estudo, utilizou-se a Revisão Integrativa (RI), método baseado em evidências, que possibilita a síntese e também a análise do conhecimento científico já produzido sobre o tema proposto (Galvão, Mendes \& Silveira, 2010). A origem do termo integrativa está relacionada com a integração de opiniões, ideias ou conceitos provindos de pesquisas utilizadas no método (Botelho, Cunha \& Macedo, 2011).

Este método de revisão amplo permite a inclusão de estudos de várias metodologias e métodos, como pesquisas experimentais e quase experimentais, tendo como objetivo uma melhor compreensão sobre o fenômeno estudado (Almeida, Lucena, Franzen \& Laurent, 2009).

Assim, a RI tem como foco colaborar para com o conhecimento investigado através de um método de pesquisa, que gera uma conclusão geral de um determinado campo de estudo de maneira sistemática (Velho, Santos, Bruggemann \& Camargo, 2012).

Considerando que a Integridade na pesquisa científica é um tema gerador de reflexões no meio acadêmico, elaborou-se uma questão norteadora para a pesquisa: Como os estudiosos vêm abordando a integridade científica em seus estudos nos diferentes contextos e regionalidades?

Para a realização da revisão, foram selecionadas as seguintes bases de dados: Literatura Latino-Americana e do Caribe em Ciências da Saúde (LILACS), Coordenação de Aperfeiçoamento de Pessoal de Nível Superior (CAPES), Scientific Electronic Library Online (Scielo) e National Library of Medicine National Institutesof Health (NLMNIH) contendo a base de dados PUBMED. A pesquisa ocorreu entre os meses de maio e julho de 2016, com coleta combinada em cada base de dados, por três pesquisadores independentes. Nas bases, utilizaram-se descritores controlados: "plagiarism and scientific misconduct and ethics research".

Como critério de elegibilidade utilizou-se artigos científicos com textos completos, originais, publicados entre 01 de janeiro de 2006 à 30 de junho de 2016, disponíveis eletronicamente, em idioma inglês, espanhol e português e que retratassem o tema de estudo. Foram excluídos os artigos duplicados encontrados nas bases de dados.Como técnica complementar, aplicou-se a busca manual de artigos científicos, também conhecida como hand search com o propósito de ampliar a confiabilidade das 
informações nas bases de dados.Os resultados da seleção estão na Figura1.

Figura 1

Fluxograma da Coleta de Dados Para RI Sobre Ética na Pesquisa Científica

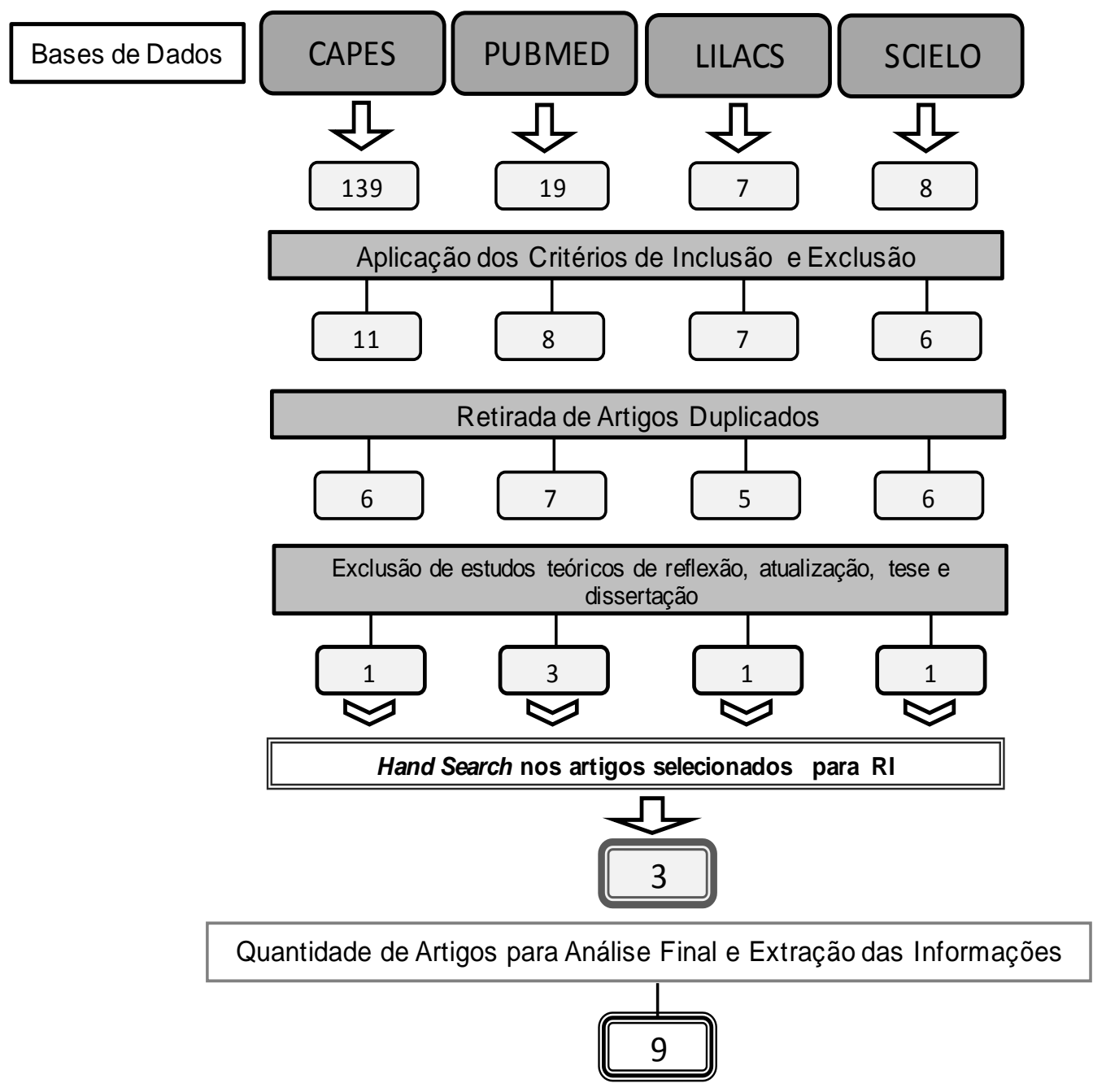

Após definir a amostra final desta revisão, utilizou-se o formulário para extração de informações de interesse, a saber: autoria, título do artigo, ano/país/base de dados/amostra, delineamento do estudo/nível de evidência/instrumentos, síntese das conclusões e resultados (Ursi \& Gavão, 2006).

O nível de evidência considerada na análise dos estudos, com classificação hierárquica, foi aplicado nos textos que preencheram os critérios de inclusão. São eles: nível I para estudos clínicos de metaanálise controlados e com randomização; 
nível II para estudo randomizado, individual e experimental; nível III para quase-experimental, não randomizado, com grupo único e controlado; nível IV para estudo não-experimental, pesquisa qualitativa ou estudo de caso; nível V para relatórios de casos ou informações obtidas sistematicamente, de qualidade verificável, ou dados de avaliação de programas e o nível VI para evidências originadas de opiniões de autoridades e/ou relatório de comitês de especialistas (Stetler e outros, 1998).

\section{Resultados}

Nesta RI analisou-se nove artigos. As extrações das informações de interesse dos artigos selecionados, quanto aos anos de publicação foram: 2007 (22\%), 2009 (33\%), 2012 (11\%), $2013(22 \%)$ e 2015 (11\%), conforme exposto na Figura 2. 
Figura 2

Informações Extraídas dos Estudos Selecionados na RI

\begin{tabular}{|c|c|c|c|c|}
\hline $\begin{array}{l}\text { Autor / } \\
\text { Ano }\end{array}$ & Título & $\begin{array}{l}\text { Delineamento do } \\
\text { estudo / Nivel de } \\
\text { evidência/Instrumentos }\end{array}$ & Resultados & Síntese das conclusőes \\
\hline $\begin{array}{l}\text { Grieger, } \\
2007\end{array}$ & $\begin{array}{l}\text { Escritores-fantasma e } \\
\text { comércio de } \\
\text { trabalhos cientificos } \\
\text { na internet: a ciéncia } \\
\text { em risco }\end{array}$ & $\begin{array}{l}\text { - Pesquisa de Campo } \\
\text { - Nivel IV } \\
\text { - Proposta de pesquisa }\end{array}$ & $\begin{array}{l}\text { Dez (55\%) responderam } \\
\text { aceitando a encomenda. } \\
\text { Seis não responderam e duas } \\
\text { na๊o aceitaram. }\end{array}$ & $\begin{array}{l}\text { A produção de trabalhos assim } \\
\text { encomendados e sua possivel } \\
\text { publicação podem tornar a literatura } \\
\text { cientifica uma fonte de informações } \\
\text { equivocadas. }\end{array}$ \\
\hline $\begin{array}{l}\text { Pryor et } \\
\text { al., } 2007\end{array}$ & $\begin{array}{l}\text { Scientific misconduct } \\
\text { from the perspective } \\
\text { of research } \\
\text { coordinators: a } \\
\text { national survey }\end{array}$ & $\begin{array}{l}\text { - Cross-sectional survey. } \\
\text {-NivelIV. } \\
\text {-the Scientific Misconduct } \\
\text { Questionnaire-Revised } \\
\text { (SMQ-R) }\end{array}$ & $\begin{array}{l}\text { It was noticed that } 18 \% \text { of } \\
\text { respondents have had contact } \\
\text { withmisconduct. }\end{array}$ & $\begin{array}{l}\text { Data supporting the impact of the } \\
\text { working environment and also on the } \\
\text { effectiveness of institutional policies } \\
\text { to reduce the scientific misconduct. }\end{array}$ \\
\hline $\begin{array}{l}\text { Fanelli, } \\
2009\end{array}$ & $\begin{array}{l}\text { How Many Scientists } \\
\text { Fabricate and Falsify } \\
\text { Research? } \\
\text { Systematic Review } \\
\text { and Meta Analysis of } \\
\text { Survey Dat a }\end{array}$ & $\begin{array}{l}\text { - Metaanalysis } \\
\text { - Nivell } \\
\text {-Survey questions on } \\
\text { plagiarism. }\end{array}$ & $\begin{array}{l}\text { The main result was the } \\
\text { percentage of respondents who } \\
\text { remembered to commit or who } \\
\text { knew a colleague commit the } \\
\text { specified behavior at least once } \\
\text { in the recall period specified. }\end{array}$ & $\begin{array}{l}\text { It appears likely that this is a } \\
\text { conservative estimate of the true } \\
\text { prevalence of scientificmisconduct. }\end{array}$ \\
\hline $\begin{array}{l}\text { Ryan et } \\
\text { al, } 2009\end{array}$ & $\begin{array}{l}\text { Undergraduate and } \\
\text { Postgraduate } \\
\text { pharmacy students' } \\
\text { perceptions of } \\
\text { plagiarism and } \\
\text { academic }\end{array}$ & $\begin{array}{l}\text { - Corte transversal } \\
\text { - Nivel IV } \\
\text { The questionnaire was } \\
\text { based on the work of } \\
\text { Carroll, Aggarwal et al, } \\
\text { Brimble and Stevenson- } \\
\text { Clarke, de Lambert et. al. } \\
\text { and Yeo. }\end{array}$ & $\begin{array}{l}\text { Widespread deficiencies in } \\
\text { student knowledge of, and } \\
\text { attitudes towards, plagiarism. } \\
\text { Students did not perceive } \\
\text { plagkism asaseriousissue. }\end{array}$ & $\begin{array}{l}\text { The study highlights the importance } \\
\text { of achieving a balance among the } 3 \\
\text { dimensions of plagiarism } \\
\text { management: prevention, detection } \\
\text { and penalty. }\end{array}$ \\
\hline $\begin{array}{l}\text { Wager et } \\
\text { al., } 2009\end{array}$ & $\begin{array}{l}\text { Science journal } \\
\text { editors' views on } \\
\text { publication ethics: } \\
\text { results of an } \\
\text { intemational survey }\end{array}$ & $\begin{array}{l}\text { - Case Study } \\
\text { - Nivel N } \\
\text { - Questionnaire } \\
\text { - StatisticalPackage for the } \\
\text { Social Sciences (SPSS) } \\
\text { version } 12.0 \\
\end{array}$ & $\begin{array}{l}\text { Responses were obtained from } \\
231 \text { editors }(44 \%) \text {, of whom } \\
48 \% \text { edited healthcare journals. }\end{array}$ & $\begin{array}{l}\text { Most editors of science joumals seem } \\
\text { not very concerned about publication } \\
\text { ethics and believe that misconduct } \\
\text { occurs only rarely in their journals. }\end{array}$ \\
\hline $\begin{array}{l}\text { Adeleye } \\
\text { \&Adebam } \\
\text { owo, 2012 }\end{array}$ & $\begin{array}{l}\text { Factors associated } \\
\text { with research } \\
\text { wrongdoing in } \\
\text { Nigeria }\end{array}$ & $\begin{array}{l}\text { - Corte transversal } \\
\text { - Nivel IV } \\
\text { - Multivariaatelogisticregre } \\
\text { ssion } \\
\end{array}$ & $\begin{array}{l}\text { Some } 22.0 \% \text { admitted to at } \\
\text { least one of fabrication, } \\
\text { falsification, and plagiarism. }\end{array}$ & $\begin{array}{l}\text { Systems that support ethical research, } \\
\text { including skilled training and funding, } \\
\text { are reco mm ended. }\end{array}$ \\
\hline $\begin{array}{l}\text { Hofmann } \\
\text { et al., } \\
2013\end{array}$ & $\begin{array}{l}\text { Scientific } \\
\text { dishonesty- a } \\
\text { nationwide survey of } \\
\text { doctoral students in }\end{array}$ & $\begin{array}{l}\text { - The study was an } \\
\text { exploratory } \\
\text { - NwellV. } \\
\text { - Anonymous questionnaire } \\
\text { in } 2010 / 2011\end{array}$ & $\begin{array}{l}79 \% \text { agreed that they would be } \\
\text { willing to report misconduct to } \\
\text { a regponsible official. }\end{array}$ & $\begin{array}{l}\text { There was little awareness of relevant } \\
\text { policies for scientific conduct, but a } \\
\text { high level of willingness to report } \\
\text { misconduct. }\end{array}$ \\
\hline $\begin{array}{l}\text { Okonta } \\
\& \\
\text { Rossouw, } \\
2013\end{array}$ & $\begin{array}{l}\text { Prevalence of } \\
\text { scientific misconduct } \\
\text { among a group of } \\
\text { researchers in }\end{array}$ & $\begin{array}{l}\text { Exploratory lesearch, } \\
\text { comenience sample of } \\
\text { lesearcheis who participated in } \\
\text { a scientific conference in } 2010 \text {. } \\
\text { - Nivelly. } \\
\text { - From the scientific } \\
\text { Misconduxt adaptation } \\
\text { Questionnaire-Pevised (SMO. } \\
\text { R). }\end{array}$ & $\begin{array}{l}\text { The plagiarism was inversely } \\
\text { associated with years of } \\
\text { research (Fisher }=p=0.02) \text {; } \\
\text { Falsification of data related to } \\
\text { the perception of low } \\
\text { effectiveness of rules } \alpha(2)= \\
6.44 \text {, p-value }=0.01) \text {; }\end{array}$ & $\begin{array}{l}\text { Training on research ethics has to be } \\
\text { integrated into the curriculum of } \\
\text { undergraduate and postgraduate } \\
\text { students while provision should be } \\
\text { made for in-service training of } \\
\text { researchers. }\end{array}$ \\
\hline $\begin{array}{l}\text { Pádua \& } \\
\text { Guilhem, } \\
2015\end{array}$ & $\begin{array}{l}\text { Integridade cientifica } \\
\text { e pesquisa em saúde } \\
\text { no Brasil: revisão da } \\
\text { literatura }\end{array}$ & $\begin{array}{l}\text { - Revisão sistemática da } \\
\text { literatura } \\
\text { - Nivel IV } \\
\text {-Preferred Reporting Items } \\
\text { for Systematic Reviews and }\end{array}$ & $\begin{array}{l}\text { Os asuntos mais discutidos } \\
\text { foram plágio e conflitos de } \\
\text { interesses sobre autoria. }\end{array}$ & $\begin{array}{l}\text { Tornase necessária a abordagem } \\
\text { precoce desses conhecimentos no } \\
\text { processo de formação acadèmica de } \\
\text { pesquisadores ejovens cientistas. }\end{array}$ \\
\hline
\end{tabular}


Observa-se uma correlação negativa entre o avanço dos anos e o número de publicação de artigos, mas não se pode afirmar que os números de publicações estão diminuindo à medida que os anos avançam. A variação do número de publicações pode sofrer influência de outras causas, sendo que no estudo consegue-se explicar somente $40 \%$ dessa variação, conforme a Figura 3.

Figura 3

Quantidade de Publicação Versus Ano Entre os Períodos 2006-2016

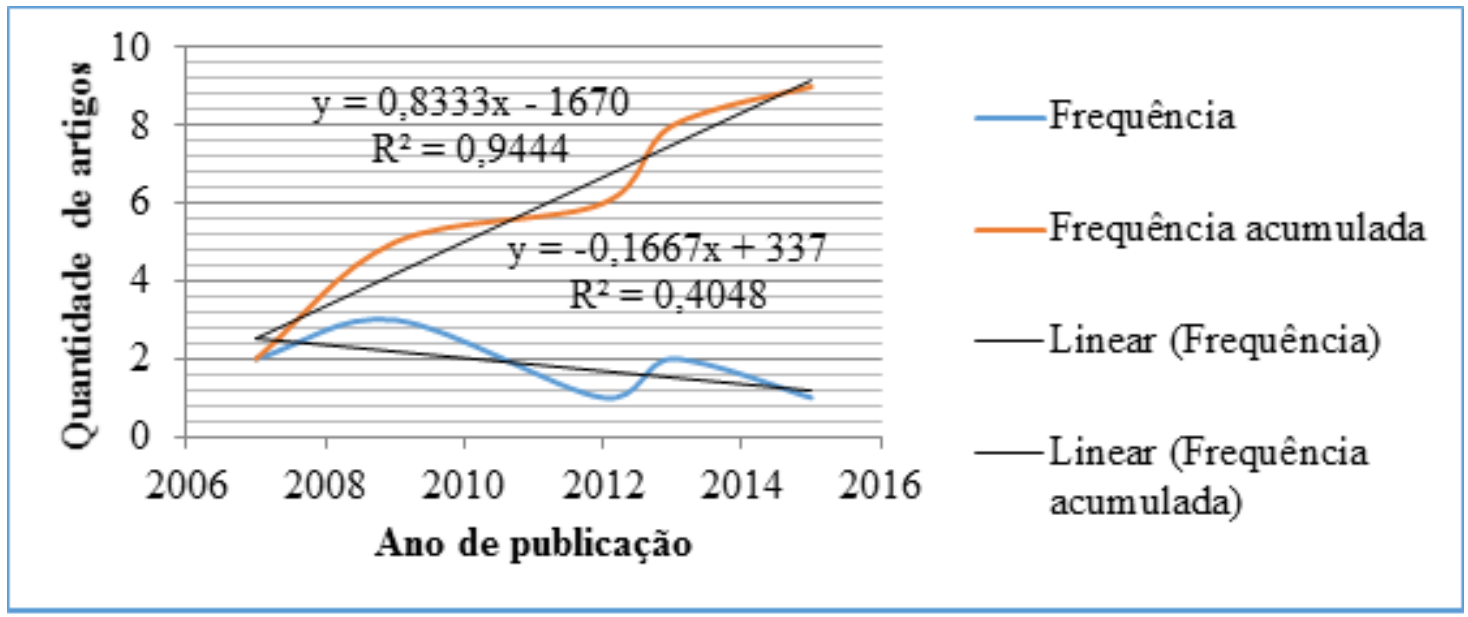

Verifica-se que o maior número de publicações está na base de dados PUBMED, que ocupa 56\%(5) dos resultados, a CAPES 22\% (2), e LILACS e Scielo compondo uma parcela de $11 \%$ (1) cada uma.

Quanto à análise do nível de evidência, $8(89 \%)$ dos artigos analisados apresentam uma abordagem qualitativa ou descritiva, com amostra de conveniência e com delineamento não experimental, caracterizando nível IV de evidência (Stetler e outros, 1998). E em 11\% (1) dos artigos identificou-se o nível I, pelo uso de meta-análise na concepção da pesquisa, de acordo de acordo com a classificação hierárquica aplicada (Stetler e outros, 1998).

Nestes estudos, constatou-se que a formação acadêmica dos alunos dentro das instituições deve incluir conhecimento sobre a má conduta científica e há uma carência de treinamentos que permitam esta reflexão (Pádua \& Guilhem, 2015; Grieger, 2007; Hofmann, Myhr \& Holm, 2013; Ryan, Bonanno, Krass, Scoullerm \& Smith, 2009; Pryor, Habermann \& Broome, 2007), além da falta de ciência das políticas institucionais (Adeleye \& Adebamowo, 2012; Ryan e outros, 2009; 
Pryor e outros, 2007) sobre a preocupação no comportamento ético.

Os estudos se originam em $44,4 \%$ (4) dos EUA, 33,3\% (3) da Inglaterra, e $22,2 \%$ (2) do Brasil, evidenciando uma maior publicação internacional referente ao tema. O campo de pesquisa das revistas que mais publicaram os artigos é da Bioética, Ciência e Medicina.

\section{Discussão}

Neste estudo, encontrou-se maior publicação científica na área das Ciências da Saúde, por ser um campo em que há muita competitividade, e também uma prática que exige a sequência de passos obrigatórios inerentes a área (Alisson, 2014). Nota-se um crescimento no ano de 2009, em virtude de Instituições de Ensino Superior (IES), adotar no ano de 2008 o uso de ferramentas tecnológicas, para detectar e tentar coibir a má conduta científica (Toledo, 2009).

As publicações que preencheram os critérios de inclusão têm origem de três países (Brasil, Inglaterra e Estados Unidos) sendo confirmada uma maior participação dos Estados Unidos nos estudos analisados, com 44,4\% dos artigos encontrados abordando integridade científica nas pesquisas. $O$ tema em questão se tornou mais evidente, especialmente neste país na década de 1980, quando revistas científicas apontaram casos de fraudes científicas em algumas importantes instituições, com o envolvimento de pesquisadores renomados (Russo, 2014). Nos países desenvolvidos, em destaque os Estados Unidos, atuam de maneira investigativa a má conduta científica, construindo uma conduta responsável de pesquisa, enquanto que em países em desenvolvimento, não possuem diretrizes e normas acadêmicas definidas sobre integridade científica (Adeleye \& Adebamowo, 2012; Heitman \& Litewka, 2011).

Embora no Brasil, a preocupação sobre integridade científica seja incipiente, vem tomando espaço em discussões, debates, Workshops e até congressos internacionais realizados, para alavancar o aprimoramento do tema. Um exemplo a ser citado é o Brazilian Meeting on Research Integrity, Science and Publication Ethics BRISPE (I Brispe, 2010; II Brispe, 2012; III Brispe, 2014) que objetiva abordar as principais práticas na condução de uma pesquisa científica e estimular o desenvolvimento das instituições do país.

Para a Coordenação de Aperfeiçoamento de Pessoal de Nível Superior (Capes) faz-se necessário a conscientização através de políticas sobre a propriedade intelectual por parte das 
instituições, sejam públicas ou privadas, suportados por recomendações do Conselho Federal da Ordem dos Advogados do Brasil (OAB). A recomendação reforça que a apropriação indevida de conteúdo de terceiros pode causar perdas imensuráveis e que a facilidade de acesso à internet é motivo de grande preocupação no sentido da prática indevida de cópias de textos. A OAB ainda aconselha a utilização de softwares antiplágio, mas ressalta que deve haver uma comissão para análise dos resultados (CAPES, 2011).

A Resolução $N^{\circ}$ 466/12 traz o esclarecimento de termos que geram dúvidas no momento da realização da pesquisa com seres humanos, fundamenta a ética científica que deve permear as atividades de pesquisa com seres humanos. Para garantir que o respeito ao ser humano será observado durante a realização da pesquisa, se faz necessária a existência do Processo de Consentimento Livre e Esclarecido que compreende as etapas de esclarecimento ao convidado a participar da pesquisa e da assinatura do Termo de Consentimento de Livre e Esclarecido (TCLE) que tem suas inserções delimitadas pela resolução.

O sistema de regulação de pesquisas com seres humanos é composto pela Comissão Nacional de Ética em Pesquisa (CONEP), pelo Conselho Nacional de
Saúde (CNS) e pelos Comitês de Ética em Pesquisa (CEP) que tem como objetivo proteger os participantes de pesquisa no Brasil. Dentro deste sistema, o CEP possui o caráter de consulta, deliberação e educação; o CONEP além das ocupações de consulta, deliberação e educação, possui também a função normativa. A análise ética da pesquisa primeiramente é encaminhada ao CEP que compete analisar e emitir parecer, após encaminha os protocolos de competência da CONEP.

Dentre outras competências, há recomendações ao pesquisador que vão além das responsabilidades éticas, como elaborar o TCLE e manter toda espécie de arquivo gerado pela pesquisa sob sua guarda durante o período mínimo de cinco anos.

Para os editores de revistas científicas, a maior inquietação é a redundância das publicações e a falta de conhecimento de potenciais problemas éticos (Wager, Fiack, Graf, Robinson \& Rowlands, 2009), o qual também existe uma controvérsia sobre o tema 'má conduta científica' e, alguns pesquisadores acreditam que, "melhorar dados", não seja uma forma de falsificação (Fanelli, 2009).

$$
\text { Os artigos encontrados }
$$
apresentaram uma frequência no que tange a má conduta científica, tais como: falsificação de dados, fabricação de dados, conflitos de interesse e com destaque para 101 
o plágio. Nota-se que, as investigações de má conduta científica, seguem a definição estabelecida em 1999, Washington, EUA, pelo Office Of Science And Technology Policy (OSTP) para má conduta científica, definindo Falsificação como qualquer forma de que descaracterize a pesquisa, sendo alteração ou omissão de partes que a compõe, manipulação de instrumentos, equipamentos ou processos da pesquisa; Fabricação são relatos, dados, registros ou resultados e o Plágio sendo a cópia de palavras ou resultados, assimilação de ideias sem fazer a referência a quem pertence (Of Science And Technology Policy [OSTP], 2000).

A prática de ghost writing (Grieger, 2007), ou seja, a não atribuição de créditos para o autor e a apropriação de sua produção, levanta o questionamento sobre a violação da ética e da moral, pois falta o compromisso deste pseudo autor para com os seus leitores. Segundo uma pesquisa realizada por um motor de busca, produziu-se mais de 4,6 milhões de referências a estes serviços em menos de meio segundo, sendo que algumas destas organizações afirmaram empregar graduados e docentes das melhores universidades para a prática de ghost writing (Singh \& Remenyi, 2016). A publicação de trabalhos por encomenda ou que apresentam falha na integridade científica, faz com que a produção científica se torne um lugar onde não se pode confiar (Gringer, 2007).

Um estudo realizado na Nigéria demonstra um índice de $68,9 \%$ de estudantes, que admitiram terem se envolvido em situações que refletem a má conduta científica (Okonta \& Rossouw, 2013), o que corrobora com um estudo realizado no Brasil, com resultado de $70 \%$ para a admissão de má conduta em situação semelhante (Oliveira, Aguiar, Queiroz \& Barrichello, 2013).

A pressão também feita ao pesquisador por uma quantidade cada vez maior de publicações para se conseguir prestígio e financiamento a pesquisa (Adeleye \& Adebamowo, 2012; Grieger, 2007; Hofmann e outros, 2013; Pryor e outros, 2007; Pádua \& Guilhem, 2015) revela ser um fator que deixa propenso o clima para que ocorra a má conduta científica ou ainda que exista uma grande quantidade de publicações de trabalhos semelhantes, fazendo com que temas relevantes, que demandam maior tempo para pesquisa não contemplem tantas produções (Camargo Jr., 2014).

Um achado nas pesquisas é que muitos autores acreditam que um relato de falta de integridade científica em uma pesquisa não seria levado a diante ou até, as experiências vivenciadas mostraram que 
o relator sofre consequências negativas ao reportar o relato ao órgão competente de investigação (Pryor e outros, 2007), o achado desencoraja as denúncias de má conduta científica, enfraquecendo o avanço na cultura responsável de pesquisa.

Outro espaço encontrado nos estudos foi em relação à quantidade de alunos por turma, onde o número de alunos tem crescido, e para que um professor faça a orientação, torna-se necessário o desenvolvimento de trabalhos em grupo, o que dificulta o acesso ao professor orientador para a construção do trabalho científico (Ryan e outros, 2009).

A Fundação de Amparo à Pesquisa do Estado de São Paulo (FAPESP) tem avaliado denúncias de má conduta científica e ao constatar violação das boas práticas, torna públicos os seus pareceres e realiza punições como suspensões do direito a solicitação de auxílios e bolsas por determinados períodos de acordo com a gravidade e natureza dos fatos (FAPESP, 2017).

$\mathrm{Na}$ Universidade Federal de Brasília (UNB), adota-se um código de ética e as denúncias passam por uma comissão constituída por três professores. Após esta avaliação, a punição vai depender do julgamento desta comissão que junto com a decisão final do reitor poderá aplicar penalidades como até a suspensão do título (UNB, 2015).
As revistas científicas, como a Revista Espaço Ética, Educação, Gestão e Consumo tem-se apoiado na legislação sobre direitos autorais $\mathrm{n}^{\circ}$ 9610, de 19 de fevereiro de 1998 e ao verificar autores que cometem plágio, realizam a suspensão de escrita em qualquer outra edição da revista.

Algumas recomendações são necessárias para coibir a má conduta científica. Um ponto de atenção para as instituições é atingir os estudantes na sua fase inicial de formação acadêmica abordando conhecimento sobre a ética e a integridade em suas produções (Pádua \& Guilhem, 2015; Hofmann e outros, 2013). Tornar a política institucional em direção a desonestidade científica mais clara e de fácil acesso para os autores, torna esta comunicação mais efetiva, diminuindo a deficiência existente em relação à má conduta e buscando uma concordância entre prevenção, detecção e penalização (Adeleye \& Adebamowo, 2012).

Outra sugestão para inibir práticas indevidas, tais como o plágio e o ghost writing, trata-se da mudança no método de avaliação nas instituições, dificultando a aquisição de produções que comprometeriam a formação acadêmica, ética e profissional dos estudantes (Okonta \& Rossouw, 2013). A participação efetiva dos orientadores é fundamental para detectar se o trabalho de um aluno é de autoria própria, pois somente na orientação 103 
é possível verificar o estilo de escrita e o nível de conhecimento (Singh \& Remenyi, 2016). E por fim, nos espaços em que a prevenção para a questão não tenha êxito, uma punição mais severa do mau comportamento seria a aplicação de penalidades tanto em níveis institucionais como nacionais (Hofmann e outros, 2013).

\section{Considerações Finais}

Este estudo buscou sistematizar o conhecimento sobre o que tange ao comportamento nas pesquisas, tal qual o plágio e a má conduta científica acometidas por uma atitude inadequada e a ética na pesquisa exercendo um papel de demonstrar a sua relevância na formação acadêmica, profissional e de caráter do indivíduo. Verificou-se que esta discussão é incipiente, pois há uma carência de padronização nos termos envolvidos que norteiam o processo de realização de pesquisa e que deixe todas as etapas explícitas, inclusive a questão da investigação em caso de má conduta na pesquisa e havendo confirmação, quais são os critérios de punição, as consequências e medidas a serem adotadas pelas instituições.

Constatou-se que o estudo pode haver limitações no uso dos critérios estabelecidos, que possivelmente restringiu a captura de algumas pesquisas abordando o tema em questão. Este fato não descaracteriza esta revisão, pois foi possível abordar o que vem sendo discutido, demonstrando que há um caminho extenso a se percorrer e a contribuir para que a ética se torne uma característica nata dos escritores nas publicações científicas.

Para enaltecer a relevância da ética nas pesquisas diante da problemática da má conduta, sugere-se uma mudança nos métodos de avaliação nas instituições, a prevenção e a punição para a redução do comportamento inapropriado com ênfase no equilíbrio entre prevenção, detecção e penalidades.

\section{Referências}

Adeleye, O. A., \& Adebamowo, C. A. (2012). Factors associated with research wrongdoing in Nigeria. Journal of Empirical Research on Human Research Ethics, 7(5), 1524.Retrieved from<http://www.ncbi.nlm.nih.gov/pubmed/23324199> DOI: 10.1525/jer.2012.7.5.15. 
Alisson, E. Má conduta científica é um problema global, afirma pesquisador. Agência Fapesp, 20 Agosto de 2014 Recuperado de $<$ http://agencia.fapesp.br/ma_conduta_cientifica_e_um_problema_global_afirma_pesq uisador/19643/>

Almeida, M., Lucena, F., Franzen, E. \& Laurent, C. M. (2009). Processo de enfermagem na pratica clínica: estudos de caso realizados no Hospital de Clínicas de Porto Alegre. Artmed Editora.

Botelho, L. L. R., Cunha, A. C. C., \& Macedo, M. (2011). O método da revisão integrativa nos estudos organizacionais. Gestão e sociedade, 5(11), 121-136.

Brazilian Meeting on Research Integrity, Science and Publications Ethics. I BRISPE Recuperado de <http://www.ibrispe.coppe.ufrj.br/>; II BRISPE, Recuperado de $<$ http://www.iibrispe.coppe.ufrj.br/>.III BRISPE, Recuperado de<http://www.wcri2015.org/in-the-news/25-iii-brispe.html>

BRASIL (2012). Resolução $\mathrm{n}^{\mathrm{o}}$ 466, de 12 de dezembro de 2012. (2013, junho 13) Regulamenta diretrizes e normas de pesquisas envolvendo seres humanos (revoga resoluções anteriores) Diário Oficial da União, Brasília: DF.

BRASIL (2011). Ministério da Ciência e Tecnologia, (2012) Estratégia Nacional de Ciência, Tecnologia e Inovação 2012-2015: Balanço das Atividades Estruturantes 2011. Brasília: Autor. Recuperado de<http://www.mct.gov.br/upd_blob/0218/218981.pdf>.

Camargo Jr, K. R. D. (2014). Publicar ou perecer, ou perecer por publicar (em excesso)?. Physis: Revista de Saúde Coletiva, 24(2), 337-339.Recuperado de<http://www.scielo.br/pdf/physis/v24n2/0103-7331-physis-24-02-00337.pdf>

CAPES (2011). Coordenação de aperfeiçoamento de pessoal de nível superior. Orientações Capes - Combate ao plágio. Brasília-DF. Recuperado de<https://www.capes.gov.br/images/stories/download/diversos/OrientacoesCapes_Com bateAoPlagio.pdf>

Cury, A. A. D. B. \& Rosing, C. K. (2013). Self-plagiarism in scientific journals: an emerging discussion. Brazilian oral research, 27(6), 451-452.Retrieved from<http://www.scielo.br/scielo.php?script=sci_arttext\&pid=S180683242013000600451> DOI: 10.1590/S1806-83242013000600001

Fanelli, D. (2009). How many scientists fabricate and falsify research? A systematic review and meta-analysis of survey data. PloS one, 4(5), e5738. Retrieved from<http://journals.plos.org/plosone/article?id=10.1371/journal.pone.0005738> DOI: 10.1371/journal.pone.0005738. 
FAPESP (2014). Fundação de Amparo à Pesquisa do Estado de São Paulo. Código de Boas Práticas Científicas. Recuperado de<http://www.fapesp.br/boaspraticas/FAPESPCodigo_de_Boas_Praticas_Cientificas_2014.pdf>

FAPESP (2017). Fundação de Amparo à Pesquisa do Estado de São Paulo. Sumários de Casos. Recuperado de<http://www.fapesp.br/8577>

Galvão, C. M., Mendes, K. D. S., \& Silveira, R. C. C. P. (2010). Revisão integrativa: método de revisão para sintetizar as evidências disponíveis na literatura. Brevidelli M.M, Sertório SCM. Trabalho de conclusão de curso: guia prático para docentes e alunos da área da saúde. São Paulo: Iátrica, 105-26.

Grieger, M. C. A. (2007). Escritores-fantasma e comércio de trabalhos científicos na internet: a ciência em risco. Ver Assoc Med Bras,53(3), 247-51.Recuperado de<http://www.scielo.br/scielo.php?script=sci_arttext\&pid=S010442302007000300023\&lang=pt> DOI: 10.1590/S0104-42302007000300023.

Heitman, E., \&Litewka, S. (2011). International perspectives on plagiarism and considerations for teaching international trainees. In Urologic Oncology: Seminars and Original Investigations (Vol. 29, No. 1, pp. 104-108). Elsevier.Retrieved from<https://www.ncbi.nlm.nih.gov/pmc/articles/PMC3038591/>. DOI: 10.1016 / j.urolonc.2010.09.014.

Hofmann, B., Myhr, A. I., \& Holm, S. (2013). Scientific dishonesty-a nationwide survey of doctoral students in Norway. BMC medical ethics, 14(1), 3. Retrieved from<http://www.biomedcentral.com/1472-6939/14/3> DOI: 10.1186/1472-6939-14-3.

Marques, F. (2014). Do Compromisso à Ação. Revista Pesquisa FAPESP, Ed. 223, 37-41. Recuperado de<http://revistapesquisa.fapesp.br/2014/09/16/compromisso-acao/>.

Oliveira, V. T. M., Aguiar, F. H., Queiroz, J. P., \& Barrichello, A. (2013). Cola, plágio e outras práticas acadêmicas desonestas: um estudo quantitativo-descritivo sobre o comportamento de alunos de graduação e pós-graduação da área de negócios. Revista de Administração Mackenzie, 15(1).

Okonta, P., \& Rossouw, T. (2013). Prevalence of scientific misconduct among a group of researchers in Nigeria. Developing world bioethics, 13(3), 149-157.Retrieved from<http://onlinelibrary.wiley.com/doi/10.1111/j.1471-8847.2012.00339.x/abstract> DOI: 10.1111/j.1471 8847.2012.00339.x.PREVALENCE.

Office Of Science And Technology Policy(2000). Federal policy on research misconduct; Preamble for research misconduct policy: Notification of final policy. Federal Register: 
06 de december de 2000, 65(235), 76260-76264. Washington, DC: Autor. Retrieved from<https://ori.hhs.gov/federal-research-misconduct-policy>.

Pádua, G. C. C., \& Guilhem, D. (2015). Integridade científica e pesquisa em saúde no Brasil: revisão da literatura. Rev. bioét. (Impr.), 23(1), 124-138. Recuperado de<http://www.scielo.br/scielo.php?script=sci_arttext\&pid=S1983$80422015000100124 \& \operatorname{lng}=$ pt\&nrm=iso\&tlng=pt> DOI: $10.1590 / 1983-80422015231053$.

Panter, M. (2015). Em suas próprias palavras: Melhores práticas para evitar o plágio. American Journal Experts. Recuperado de<https://www.aje.com/assets/docs/AJEPlagarism-Best-Practices-Portuguese-2015.pdf>.

Pithan, L. H., \& Oliveira, A. P. (2013). Ética e integridade na pesquisa: o plágio nas publicações científicas. $\operatorname{Rev} \quad$ AMRIGS, 57(3), 240-5. Recuperado de<http://www.amrigs.com.br/revista/57-03/1250.pdf>

Pryor, E. R., Habermann, B., \& Broome, M. E. (2007). Scientific misconduct from the perspective of research coordinators: a national survey. Journal of Medical Ethics, 33(6), 365-369.Retrieved

from<http://www.ncbi.nlm.nih.gov/pmc/articles/PMC2598278/pdf/365.pdf> $\quad$ DOI: 10.1136/jme.2006.016394.

Russo, M. (2014). Ética e integridade na ciência: da responsabilidade do cientista à responsabilidade coletiva. Estudos Avançados, 28(80), 189-198.

Ryan, G., Bonanno, H., Krass, I., Scouller, K., \& Smith, L. (2009). Undergraduate and postgraduate pharmacy students' perceptions of plagiarism and academic honesty. American journal of pharmaceutical education,73(6), 105.Retrieved from<http://www.ncbi.nlm.nih.gov/pmc/articles/PMC2769527/>DOI: 10.5688/aj7306105.

Singh, S., \& Remenyi, D. (2016). Plagiarism and ghostwriting: The rise in academic misconduct. South African Journal of Science, 112(5-6), 1-7.

Stetler, C. B., Morsi, D., Rucki, S., Broughton, S., Corrigan, B., Fitzgerald, J., ...\& Sheridan, E. A. (1998). Utilization-focused integrative reviews in a nursing service. Applied $\begin{array}{llll}\text { Nursing } & \text { Research, 11(4), 195-206. } & \text { Retrieved }\end{array}$ from<http://www.ncbi.nlm.nih.gov/pubmed/9852663>.

Suzuki, E. (2015, dezembro 10). UnB promove ações de combate ao plágio.Unb Notícias. Recuperado de <http://noticias.unb.br/publicacoes/112-extensao-e-comunidade/212-unbpromove-acoes-de-combate-ao-plagio> 
FABIANA DIVINA DA SILVA, ROBERTA ALMEIDA ELIAS, SULAMITA DA SILVA LUCAS, ROSELMA LUCCHESE, IVÂNIA VERA

Toledo, K.(2009). Universidades recorrem a software contra plágio. O Estado de São Paulo. Recuperado

de<http://www.ufcg.edu.br/prt_ufcg/assessoria_imprensa/mostra_noticia.php?codigo=85 91>

Universidade de Brasília (2015). UnB promove ações de combate ao plágio. Recuperado de $<$ http://noticias.unb.br/publicacoes/112-extensao-e-comunidade/212-unb-promove-acoesde-combate-ao-plagio>

Ursi, E. S., \& Gavão, C. M. (2006). Prevenção de lesões de pele no perioperatório: revisão integrativa da literatura. Revista Latino-Americana de Enfermagem, 14(1), 124-131.

Velho, B. M., Santos, K. A. E., Brüggemann, O. M., \& Camargo, V. B. (2012). Vivência do Parto Normal ou Cesáreo: Revisão Integrativa Sobre a Percepção de Mulheres. Texto \& Contexto Enfermagem,21(2). Recuperado de< http://dx.doi.org/10.1590/S010407072012000200026> DOI: 10.1590/S0104-07072012000200026.

Wager, E., Fiack, S., Graf, C., Robinson, A., \& Rowlands, I. (2009). Science journal editors' views on publication ethics: results of an international survey. Journal of medical ethics, 35(6), 348-353.Retrieved from<http://jme.bmj.com/content/35/6/348.full.pdf> DOI: $10.1136 /$ jme.2008.028324.

\section{As Autoras:}

Fabiana Divina da Silva, graduada em Matemática pela Universidade Federal de Goiás (UFG). Email: fabianadivsilva@gmail.com

Roberta Almeida Elias, graduada em Redes de Comunicação pelo Centro Federal de Educação Tecnológica do Estado de Goiás (CEFET). Email: robertaellias@gmail.com

Sulamita da Silva Lucas, graduada em Administração pela Universidade Federal de Goiás (UFG). Email: sulamita-lucas@hotmail.com

Roselma Lucchese, Enfermeira. Prof Associada IBiotec/UFG/RC. docente no Programa de mestrado Gestão Organizacional. roselmalucchese@ hotmail.com

Ivânia Vera, Enfermeira. Prof. Associada IBiotec/UFG/RC. docente no Programa de mestrado Gestão Organizacional. ivaniavera@gmail.com

Recebido em: 27/04/2017.

Aprovado em: 19/06/2017. 